\title{
Radiofrequency ablation for treatment of medically inoperable stage I non-small cell lung cancer
}

\author{
Michael Lanuti, MD, ${ }^{\mathrm{a}}$ Amita Sharma, MD, ${ }^{\mathrm{b}}$ Subba R. Digumarthy, MD, ${ }^{\mathrm{b}}$ Cameron D. Wright, MD, ${ }^{\mathrm{a}}$ Dean M. Donahue, MD,

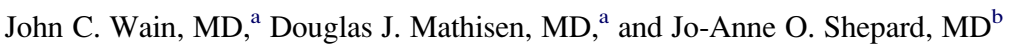

\begin{abstract}
Objective: This study evaluated long-term results of radiofrequency ablation for medically inoperable earlystage lung cancer.

Methods: Thirty-one consecutive patients with biopsy-proven non-small cell lung cancer underwent 38 treatments of computed tomographically guided radiofrequency ablation in a 4.5-year period. All patients were carefully selected and deemed medically ineligible for resection by a multidisciplinary team. Radiofrequency ablation was performed with curative intent with a single or cluster cool-tip electrode. Patients were hospitalized for 23-hour observation.
\end{abstract}
Results: Treatment was complete in all cases, with no 30-day mortality. Local recurrence was confirmed radio- graphically by computed tomography, positron emission tomography, or both after $31.5 \%$ of treatments (12/38). Two patients were successfully retreated for technical failures related to pneumothorax; 3 underwent radiotherapy with stable disease. Mean maximal diameter of 38 tumors treated was $2.0 \pm 1.0 \mathrm{~cm}$ (range $0.8-4.4 \mathrm{~cm}$ ). After median follow-up of $17 \pm 11$ months, $74 \%$ of patients $(23 / 31)$ were alive. Three patients died of metastatic dis- ease; 5 died of pneumonia remote from treatment. The 2- and 4-year survivals were $78 \%$ and $47 \%$, respectively. Median overall survival was 30 months. Pneumothorax (13\%), pneumonia (16\%), and pleural effusion (21\%), were the most common complications.

Conclusions: Radiofrequency ablation of medically inoperable early-stage lung cancer in carefully selected patients yields encouraging midterm results without significant loss of pulmonary function. Local tumor progression appears related to lung tumors larger than $3 \mathrm{~cm}$. Computed tomography and positron emission tomography need further validation for the early identification of local tumor progression following radiofrequency ablation.

\begin{abstract}
Alternative therapy for unresectable stage I non-small cell lung cancer (NSCLC) has historically been conventional external beam radiotherapy (XRT), with only modest longterm local control. ${ }^{1,2}$ In a meta-analysis of 26 nonrandomized studies treating inoperable stage I or II NSCLC with high-dose XRT, 3-year survival and local failure rates were $17 \%$ to $55 \%$ and $6 \%$ to $70 \%$, respectively. ${ }^{3}$ Traditional surgical therapy for early-stage lung cancer may not be feasible because of significant cardiopulmonary compromise or a patient's refusal of surgical intervention. Newer nonsurgical modalities include bioimage-guided 4-dimensional radiation, stereotactic radiotherapy, ${ }^{4}$ and lung radiofrequency ablation (RFA). There has been accumulating experience from a number of investigators that lung RFA is safe and feasible for the treatment of unresectable stage I lung cancer. ${ }^{5-9}$ Limitations of this technology for solid
\end{abstract}

From the Divisions of Thoracic Surgery ${ }^{\mathrm{a}}$ and Thoracic Radiology, ${ }^{\mathrm{b}}$ Massachusetts General Hospital, Harvard Medical School, Boston, Mass.

Read at the Eighty-eighth Annual Meeting of The American Association for Thoracic Surgery, San Diego, Calif, May 10-14, 2008.

Received for publication May 2, 2008; revisions received Aug 17, 2008; accepted for publication Aug 21, 2008.

Address for reprints: Michael Lanuti, MD, Division of Thoracic Surgery, Massachusetts General Hospital, 55 Fruit St, Blake 1570, Boston, MA 02114 (E-mail:

Mlanuti@partners.org).

J Thorac Cardiovasc Surg 2009;137:160-6

$0022-5223 / \$ 36.00$

Copyright (c) 2009 by The American Association for Thoracic Surgery

doi:10.1016/j.jtcvs.2008.08.034 tumor ablation in the lung are tumor size and proximity to blood vessels.

Because there have been no randomized, controlled trials assessing long-term outcome of newer radiation techniques versus RFA for lung cancer, these modalities must be carefully scrutinized. There is no standardization of lung RFA, and many of the reported series used different RFA systems. The clinical efficacy of tumor destruction with different RFA systems has not been formally compared. Regional disease progression is often poorly characterized in contemporary series, because hilar and mediastinal lymph nodes are evaluated by imaging studies and not pathologic verification.

In this study, we evaluated the outcomes of stage I NSCLC treated with a single RFA system and highlighted local control, patterns of failure, complications, and changes in pulmonary function. Positron emission tomography (PET) was also implemented in the detection of local tumor progression.

\section{MATERIALS AND METHODS \\ Subject Selection}

The study population included consecutive patients with NSCLC undergoing lung RFA from July 2003 to February 2008 at the Massachusetts General Hospital. All participants were evaluated by a multidisciplinary team including representatives from medical oncology, radiation oncology, thoracic surgery, and thoracic radiology. Patients with NSCLC had clinical staging with chest computed tomography (CT) and PET scan within 60 days of evaluation. Patients with fluorodeoxyglucose-avid mediastinal 


\section{Abbreviations and Acronyms \\ $\mathrm{CT}=$ computed tomography \\ NSCLC $=$ non-small cell lung cancer \\ PET = positron emission tomography \\ RFA $=$ radiofrequency ablation \\ XRT $=$ external beam radiotherapy}

TABLE 1. Patient and tumor characteristics $(\mathbf{N}=\mathbf{3 1})$

\begin{tabular}{|c|c|}
\hline Sex (female/male) & $17: 14$ \\
\hline Age $(y$, median $)$ & 70 \\
\hline \multicolumn{2}{|l|}{ Lesion size $(\mathrm{cm})$} \\
\hline Mean \pm SD & $2.0 \pm 1.0$ \\
\hline Range & $0.8-4.4$ \\
\hline Previous lung cancer (No.) & $17(55 \%)$ \\
\hline \multicolumn{2}{|l|}{ Zubrod score (No.) } \\
\hline 0 & 9 \\
\hline 1 & 14 \\
\hline 2 & 7 \\
\hline 3 & 1 \\
\hline \multicolumn{2}{|l|}{ Anesthesia } \\
\hline Conscious sedation & 24 \\
\hline General & 7 \\
\hline \multicolumn{2}{|l|}{ Clinical stage ( $\mathrm{N}=34$ tumors $)$} \\
\hline T1N0 & 29 \\
\hline T2NO & 5 \\
\hline \multicolumn{2}{|l|}{ Histologic type } \\
\hline \multicolumn{2}{|l|}{ Squamous } \\
\hline No. & 4 \\
\hline Mean tumor size (cm) & 2.9 \\
\hline \multicolumn{2}{|l|}{ Adenocarcinoma } \\
\hline No. & 20 \\
\hline Mean tumor size (cm) & 1.9 \\
\hline \multicolumn{2}{|l|}{ Bronchoalveolar carcinoma } \\
\hline No. & 10 \\
\hline Mean tumor size $(\mathrm{cm})$ & 1.9 \\
\hline
\end{tabular}

lymph nodes or lymph nodes larger than $1.0 \mathrm{~cm}$ in short-axis diameter were evaluated by mediastinoscopy. Medical inoperability was determined by a thoracic surgeon according to advanced cardiopulmonary morbidities: (1) compromised pulmonary function, measured as forced expiratory volume in 1 second less than $40 \%$ predicted, diffusion capacity of carbon monoxide less than $50 \%$ predicted, or oxygen desaturation with climbing one flight of stairs; (2) severe vascular disease; (3) high cardiac risk, including unstable angina or ejection fraction less than $30 \%$; and (4) poor performance status (Zubrod score of 2 to 3). Patients with PET-positive or enlarged N1 nodes within the same lobe of the lesion were excluded from RFA treatment. Inclusion criteria for lung RFA were as follows: tumors that were medically inoperable, tumor size smaller than $4 \mathrm{~cm}$, no evidence of extraregional (outside of the involved lobe) disease, or patient refusal of surgery because of elevated risk profile. Before lung RFA, all lesions underwent biopsy with CT-guided fine-needle aspiration to confirm the diagnosis. RFA treatment was performed at a separate setting after informed, written consent had been obtained. Approval for this retrospective study was granted by the institutional review board at the Massachusetts General Hospital.

\section{RFA Treatment}

All lung RFA treatments were performed percutaneously with a single or cluster cool-tip electrode coupled to a generator and perfusion pump (Covidien, Mansfield, Mass). Procedures were performed with CT guidance (64-slice CT; Siemens Corp, New York, NY) with 2.5-mm collimation. Although some patients received general anesthesia because of severe cardiac or pulmonary compromise, conscious sedation with local anesthesia was the preferred method. The cluster electrode was implemented for lesions larger than $1 \mathrm{~cm}$, whereas the single electrode was used for lesions $1 \mathrm{~cm}$ or smaller. Once in place, a 12-minute RFA treatment was initiated, during which the patient was closely monitored for adequate pain control, respiration, and oxygenation. After this time, temperature within the lesion was measured to achieve more than $60^{\circ} \mathrm{C}$ (usually about $90^{\circ} \mathrm{C}$ ) for the ablation to be considered adequate. Before the electrode was removed, a CT scan was performed to assess for an adequate margin of treatment, which was seen as a ground-glass opacity surrounding the tumor, and to detect any immediate complications, such as hemothorax, pneumothorax, or pulmonary hemorrhage. If necessary, the electrode was repositioned and an additional treatment performed.

Patients were admitted overnight for 23-hour observation and prescribed oral analgesic narcotics to treat any pleuritic pain after the procedure. In addition, prophylactic antibiotics were administered to patients with prosthetic cardiac valves, pacemakers, mitral valve prolapse, or joint prostheses. $\mathrm{Pa}-$ tients were instructed to watch for the onset of fever or sputum production, which could signal the development of pneumonia.

Pulmonary function was routinely measured a few months before scheduled RFA and then 3 to 6 months after treatment. Imaging follow-up was performed to assess for treatment efficacy and local tumor progression. PET with diagnostic quality CT was performed after 1, 6, 12, and 24 months to help define its role in the early identification of local tumor progression. Additional CT scans were obtained at 3, 9, and 18 months. If there was evidence of disease progression, repeated RFA was considered.
Local control was defined as lack of focal or diffuse enlargement of the ablated lesion on CT and no evidence of eccentric enhancement on PET at a minimum of 3 months of follow-up. Progression of fluorodeoxyglucoseavid regional lymphadenopathy (hilar or mediastinal) after 3 months was also considered a local failure.

\section{Statistical Methods}

Survival curves for overall, disease-free, and local tumor progressionfree survivals were constructed with the Kaplan-Meier method and compared with the log rank test. Continuous variables were analyzed with the Student $t$ test and confirmed with the Mann-Whitney $U$ test. The statistical software package SPSS 14.0 (SPSS Inc, Chicago, Ill) was used for all survival analyses.

\section{RESULTS}

Thirty-one patients with 34 tumors underwent 38 lung RFA treatments for clinical stage I NSCLC from July 2003 to February 2008. The mean maximal tumor diameter was $2.0 \pm 1.0 \mathrm{~cm}$ (range $0.8-4.4 \mathrm{~cm}$ ). Other patient characteristics are described in Table 1 . The majority of lung tumors were adenocarcinomas, clinical T1N0. More than half of the patients had a remote history of resected NSCLC, contributing to their limited pulmonary function. Three patients had more than 1 tumor considered to be synchronous or metachronous primary lung cancers.

Inoperability was determined by clinical parameters or the patients desire to avoid surgery in association with their medical comorbidities. Limited pulmonary function was 
TABLE 2. Summary of complications for 38 treatments

\begin{tabular}{lcr}
\hline & No. & $\%$ \\
\hline Pneumothorax & 5 & 13 \\
Chest tube insertion & 3 & 8 \\
Minor hemoptysis & 6 & 16 \\
Hemothorax & 2 & 5 \\
Pneumonia (within 30 d) & 6 & 16 \\
Pleural effusion & 8 & 21 \\
Neuropathy & 1 & 3 \\
Bronchopleural fistula & 3 & 8 \\
\hline
\end{tabular}

found to be the primary determinant of medical unresectability in 17 patients $(55 \%)$. Eleven patients $(35 \%)$ with medical comorbidities and preserved pulmonary function refused surgery because of elevated surgical risk. Three patients $(10 \%)$ had significant cardiac or vascular disease that put them at high risk with surgical intervention.

Pneumonia, pneumothorax, minor hemoptysis, and pleural effusion were the most common complications, as listed in Table 2. All pneumonias occurred within 4 weeks of ablation and resolved with a course of oral antibiotics. Peripheral cavities with air-fluid levels (representing limited bronchopleural fistula) were observed in 3 patients; these ultimately resolved with observation. One patient had transient right recurrent laryngeal nerve palsy as a result of thermal injury from treating a right upper lobe tumor in proximity to the anterior mediastinum. There was no major episodes of hemoptysis or pulmonary hemorrhage in this series.

Local tumor progression after RFA was observed in $31.5 \%$ of treated tumors $(12 / 38)$. The average tumor size in patients with local treatment failure was $2.3 \pm 1.0 \mathrm{~cm}$, versus $1.8 \pm 0.9$ $\mathrm{cm}$ in those with no recurrence $(P=.15)$. Tumors larger than $3.0 \mathrm{~cm}$ had the highest local recurrence rate $(50 \%)$, whereas tumors smaller than $2.0 \mathrm{~cm}$ had the lowest (Table 3). Seventyfive percent of the tumors that had local failure $(9 / 12)$ were recognized within 6 months of the original treatment. Two patients were successfully retreated early for technical failure related to procedure-related pneumothorax. Three patients had local progression that subsequently responded to XRT with stable disease. Another patient with a slow-growing lung cancer, who had initially refused surgery after previous pulmonary resections, had local recurrence 17 months after RFA and underwent a salvage right upper lobectomy. His final pathologic examination showed a $1-\mathrm{cm}$ residual adenocarcinoma (T1N0M0) with extensive hyaline fibrosis, consistent with treatment effect. Ninety percent of patients with a diagnosis of bronchoalveolar carcinoma (9/10, mean tumor size

TABLE 3. Summary of local tumor progression by size

\begin{tabular}{lcc}
\hline Tumor size (cm) & No. of lesions & Local recurrence (\%) \\
\hline $0-1.9$ & 23 & $21.7 \%$ \\
$2.0-3.0$ & 9 & $44.4 \%$ \\
$>3.0$ & 6 & $50.0 \%$ \\
\hline
\end{tabular}

TABLE 4. Characteristics of 34 lesions treated with lung radiofrequency ablation

\begin{tabular}{lc} 
Treatments (No.) & 38 \\
Repeat treatments (No.) & 4 \\
Local failure (No.) & $12(31.5 \%)$ \\
Alive (No.) & $23(74 \%)$ \\
Disease free (No.) & $17(74 \%)$ \\
Disease progression (No.) & $6 / 23(26 \%)$ \\
Follow-up (mo, median \pm SE) & $17.3 \pm 11$ \\
Overall survival (mo, median \pm SE) & $30 \pm 1.5$ \\
Local recurrence-free survival (mo, mean $\pm \mathrm{SE})$ & $33 \pm 3.8$ \\
\hline
\end{tabular}

$1.9 \pm 0.6 \mathrm{~cm}$ ) showed no evidence of local tumor progression at a median follow-up of $10.5 \pm 15$ months.

Treatment was successfully performed in all cases with no 30-day mortality. Seventy-four percent of patients (23/ 31 ) were alive after a median follow-up of $17.3 \pm 11$ months (Table 4). Five patients died of respiratory complications (pneumonia) related to chronic obstructive pulmonary disease, and 3 died of disseminated progression of lung cancer. The overall 2- and 3-year survivals (Figure 1) were $78 \%$ and $47 \%$, respectively. Median overall and disease-free survivals were 30 months and 25.5 months, respectively. Disease-free survivals (Figure 2) were $57 \%$ at 2 years and $39 \%$ at 3 years. Mean local tumor progression-free survival was $33 \pm 3.8$ months (Figure 3). Median local tumor progression-free survival was not reached. Tumors smaller than $2 \mathrm{~cm}$ were associated with better local progressionfree survival than were larger tumors (Figure 4), although not statistically significantly so $(P=.213)$.

Pulmonary function was evaluated in 23 patients before and 3 to 6 months after treatment (Table 5). There was no difference in both measured forced expired volume at $1 \mathrm{sec}-$ ond and diffusion capacity.

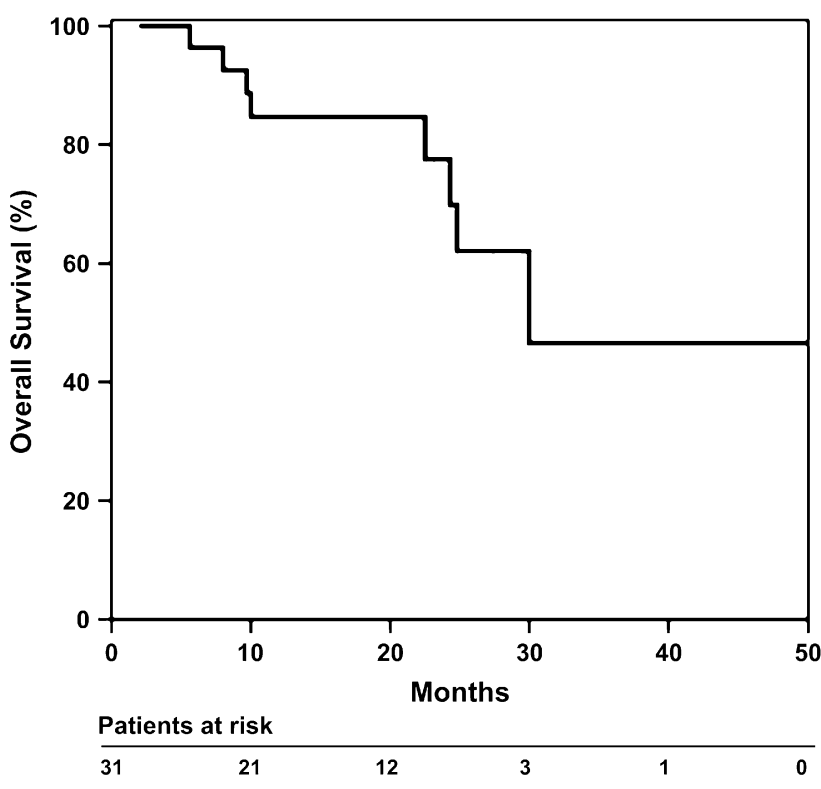

FIGURE 1. Overall survival (1-year 85\%, 2-year 78\%, 3-year 47\%). 


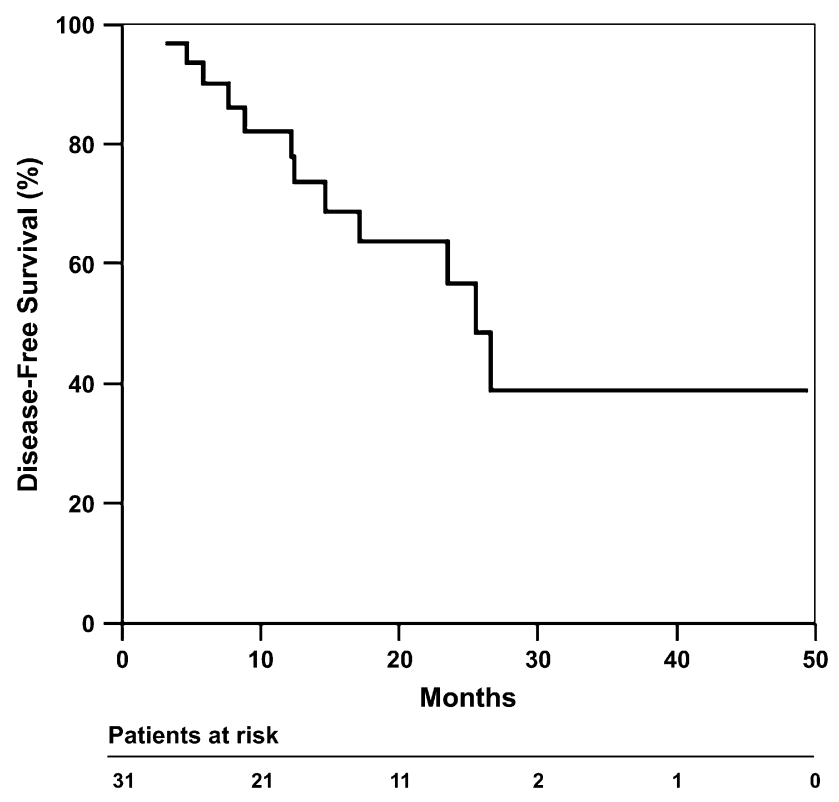

FIGURE 2. Disease-free survival (1-year 82\%, 2-year 57\%, 3-year 39\%).

\section{DISCUSSION}

Surgical resection remains the mainstay for early-stage NSCLC and provides the best chance for cure. ${ }^{10,11}$ Highrisk patients with limited cardiopulmonary reserve or poor performance status, however, require alternative treatment strategies. The major advantages of RFA therapy for the treatment of medically unresectable stage I NSCLC are low morbidity, single application, reduced hospital stay, and well-defined zones of tissue destruction. In contrast, conventional radiotherapy and stereotactic radiotherapy

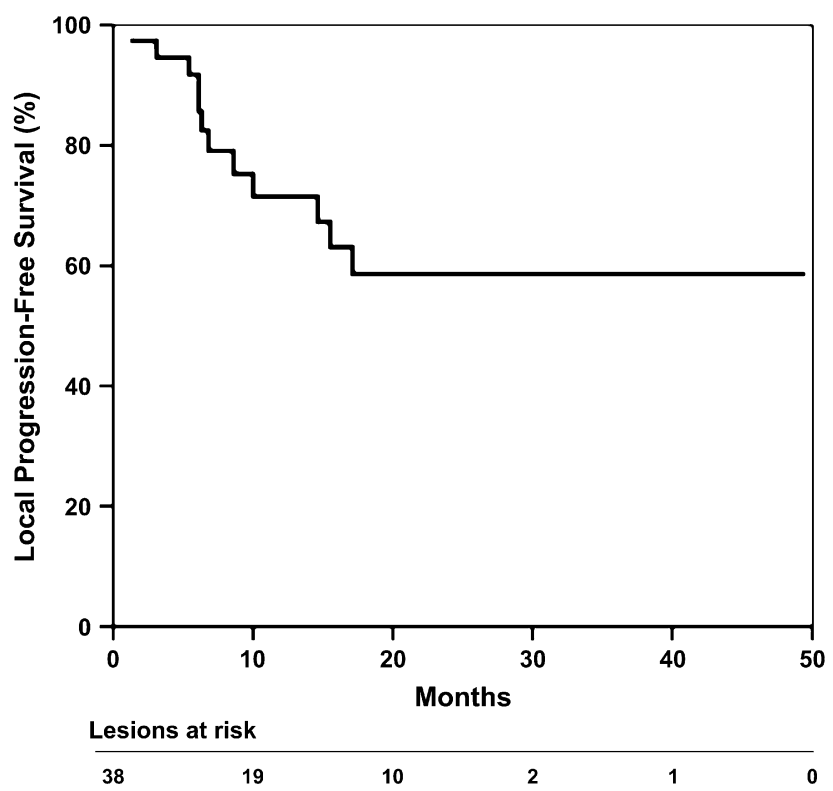

FIGURE 3. Local progression-free survival (1-year $71 \%$, 2-year $58 \%$, 3-year 58\%).

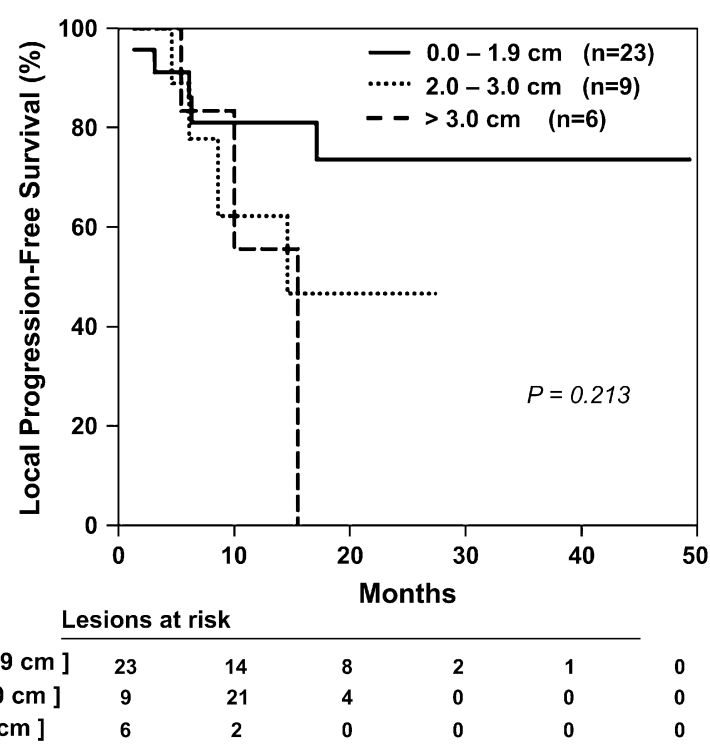

FIGURE 4. Local progression-free survival stratified by tumor size.

require multiple treatments and are often associated with pneumonitis, bronchial stenosis, esophagitis, and complications of fiducial markers. Long-term outcomes of stereotactic radiotherapy in North America and Japan are still being accumulated, but some reports indicate 2-year local control rates of $63 \%$ to $95 \%$ (2-year survival about $55 \%$ ) with high-dose fractions but significant late toxicity, reported as radiation pneumonitis, particularly in central tumors. ${ }^{4,12-14}$

In a recent review of 17 studies examining case series of RFA for lung tumors, Zhu and associates ${ }^{15}$ highlighted many of the challenges that make comparisons and data analysis difficult. Aside from the implementation of different RFA systems, there is no uniformity in RFA treatment responses, patient populations and tumor characteristics are heterogeneous, the definitions of local tumor progression and radiologic assessment are variable, and patient followup remains short. Fernando and colleagues ${ }^{5}$ attempted to standardize treatment response by using modified response evaluation criteria in solid tumors (RECIST), but these criteria have not been widely accepted in RFA literature. In our

TABLE 5. Measured pulmonary function in 23 patients after lung ablation

\begin{tabular}{lcc}
\hline \multicolumn{1}{c}{ Parameter } & \multicolumn{1}{c}{ Preablation } & Postablation (3-6 mo) \\
\hline $\begin{array}{l}\text { Forced expiratory } \\
\quad \text { volume in 1 s (L, mean } \pm \mathrm{SD})\end{array}$ & $1.58 \pm 0.71$ & $1.59 \pm 0.66^{*}$ \\
$\begin{array}{l}\text { Percentage of predicted } \\
\quad \text { forced expiratory volume }\end{array}$ & $62.2 \% \pm 25 \%$ & $61.4 \% \pm 25 \% \dagger$ \\
$\quad$ in 1 s (mean $\pm \mathrm{SD})$ & & \\
Percentage of predicted & $56 \% \pm 25 \%$ & $55.8 \pm 22 \%$ \\
$\quad$ carbon monoxide lung & & \\
$\quad$ diffusion capacity (mean $\pm \mathrm{SD})$ & & \\
*By $t$ test, $P=.39 . \dagger$ By $t$ test, $P=.26$. &
\end{tabular}


experience, detection of local tumor progression was enhanced by comparing PET characteristics of the ablated lesion at 1,6 , and 12 months (the time at which $75 \%$ of the lesions in this study had local failure). Characteristics of local failure were defined by eccentric activity on PET or lack of expected contraction of the ablated zone on chest CT over time.

The American College of Surgeons is sponsoring a multiinstitutional phase I study (ASOSOG Z4033) to address uniformity of RFA treatment in high-risk patients with stage IA NSCLC. Primary objectives are to establish 2-year survival, to assess freedom from local and regional recurrence, to assess the role of PET in predicting local control, and to explore short- and long-term effects of RFA on pulmonary function. Although we were unable to evaluate pulmonary function in our entire study cohort, this report is the first to demonstrate no statistically discernible difference in lung spirometry or diffusion capacity ( $\mathrm{N}=23)$ at 3 to 6 months after ablation. Despite higher than expected mean values for percentage predicted forced expiratory volume in 1 second $(62 \%)$ and diffusion capacity of carbon monoxide $(56 \%)$ in this patient series, many lesions were considered medically unresectable because of cardiac comorbidities (eg, critical aortic stenosis, coronary artery disease, cardiomyopathy), and $35 \%$ of patients refused surgery. Patients were selected for RFA on the basis of anatomic criteria, mostly influenced by peripheral location and size $3 \mathrm{~cm}$ or smaller. Central lesions were more likely to be referred for radiation therapy. The authors found that referrals for RFA were also partly patient driven, with high-risk patients favoring a single treatment modality rather than multiple treatments.

In studies that treated early-stage NSCLC with RFA, local tumor progression ranged from $3 \%$ to $42 \% .{ }^{6,7,16,17}$ We observed a $31.5 \%$ local tumor progression rate at 17 months after RFA. Factors that have been reported to influence local failure include tumor size; proximity to pulmonary vessels, aorta, or azygous vein, which is associated with a "heat sink" effect; and a satisfactory margin of ablation at least $1 \mathrm{~cm}$ beyond the edge of tumor. The importance of ablation beyond the tumor margin was eloquently evaluated by separate investigators with porcine and rabbit models of lung RFA. ${ }^{18,19}$ These investigators demonstrated that there were three concentric histologic zones of ablation (central, intermediate, and peripheral) with mixed populations of cells. The central and intermediate zones contained nonviable cells, whereas the peripheral edge of ablation $(2-5 \mathrm{~mm})$ contained both necrotic and viable cells, suggesting the technical need for RFA well beyond the edge of tumor. These investigators also reported that the ground-glass attenuation observed on CT corresponded to all three zones and therefore would overestimate the diameter of complete necrosis. Limitations of RFA technology for lung neoplasm can potentially be addressed with combined modality treatment (RFA followed by XRT), because central tumor hypoxia responds better to ablation. ${ }^{20}$
There was no 30-day mortality in our patient series, and there were no complications requiring prolonged hospitalization or significant additional medical intervention. Although pleural effusions were relatively common $(21 \%)$, none required drainage. Pneumothorax in this series was less common $(13 \%)$ than in other published series, which is potentially attributable to pleural symphysis as a result of previous lung surgery in about $50 \%$ of the patients. Transient recurrent laryngeal nerve palsy, as observed in 1 patient after upper lobe RFA, has been previously described. ${ }^{21}$

Because many patients with medically unresectable stage I NSCLC have severe comorbidities, overall survival after lung RFA is an inappropriate surrogate for treatment success. Only 3 of the 8 patients in this study who died did so of complications related to malignant disease progression, whereas the remaining 5 of 8 died of complications of their medical comorbidities remote to the time of RFA ablation, emphasizing the compromised medical condition of these selected patients. Freedom from local and regional progression is a far superior method of analyzing RFA outcome. We observed estimated 3-year progression-free and disease-free survivals of $58 \%$ and $39 \%$, respectively, at a median follow-up of 17 months (range 3-35 months). Although direct comparison is difficult with small patient numbers, limited or sublobar resection in compromised patients for T1N0 NSCLC in North America and Europe has a 3-year disease free survival of about $60 \%{ }^{11,22}$ RFA for stage I NSCLC is still considered a compromise and should not be substituted for anatomic surgical resection, which currently offers the best long-term survival.

Patterns of local or systemic recurrence after RFA of solid tumors have not been thoroughly evaluated in published series. Acceleration of metastatic disease after liver RFA has been considered, ${ }^{23}$ but this phenomenon has not been studied in the lung RFA experience. In an experience of more than 50 RFA treatments of solid tumors in lungs (even in patients with post-RFA pleural effusions), we have not documented any pleural dissemination of metastatic disease with the Covidien probe. The patterns of extraregional disease progression (listed in Table 6) are commonly seen in the treatment of primary lung cancer, but no conclusions can be derived regarding acceleration of malignant disease

TABLE 6. Pattern of disease progression after radiofrequency ablation

\begin{tabular}{lc}
\hline \multicolumn{1}{c}{ Metastatic site } & No. \\
\hline Ipsilateral lung & 3 \\
Contralateral lung with multifocal bronchoalveolar carcinoma & 3 \\
Liver & 3 \\
Bone & 3 \\
Brain & 1 \\
Ipsilateral regional lymph nodes & 2 \\
\hline
\end{tabular}


in this study without a matched surgical cohort undergoing sublobar resection.

We recognize that this is a prospective, observational study with limited patient numbers and relatively short follow-up. This is also a highly selected patient population subject to the institutional biases of the multidisciplinary team. Unlike previous series, we did not use multiple RFA systems to deliver treatments. Because 3 patients received conventional XRT to lesions for which RFA had failed, firm conclusions regarding outcome in these cases is confounded.

In summary, experience with RFA for stage I NSCLC is accumulating. Intermediate results are encouraging and rival the local control observed with conventional XRT. As newer noninvasive alternatives emerge for medically inoperable tumors, our ability to accurately stage cancers becomes even more critical. One should emphasize the need for thoracic surgeons to be involved in the clinical decisions for these difficult cases. Invasive staging modalities, such as mediastinoscopy and endobronchial or endoesophageal ultrasonography should be used for biopsy of suspect hilar or mediastinal lymph nodes. Determination of response to treatment and detection of local failure require careful evaluation with anatomic (diagnostic CT) and functional (PET) imaging by institutions experienced with RFA.

We acknowledge our research coordinators, Emily Nohrden, $\mathrm{MPH}$, and JoAnne Martino, RN, for compiling some of the data.

\section{References}

1. Qiao X, Tullgren O, Lax I, Sirzen F, Lewensohn R. The role of radiotherapy in treatment of stage I non-small cell lung cancer. Lung Cancer. 2003;41: $1-11$.

2. Sibley GS, Jamieson TA, Marks LB, Anscher MS, Prosnitz LR. Radiotherapy alone for medically inoperable stage I non-small-cell lung cancer: the Duke experience. Int J Radiat Oncol Biol Phys. 1998;40:149-54.

3. Rowell NP, Williams CJ. Radical radiotherapy for stage I/II non-small cell lung cancer in patients not sufficiently fit for or declining surgery (medically inoperable): a systematic review. Thorax. 2001;56:628-38.

4. Onishi H, Araki T, Shirato H, Nagata Y, Hiraoka M, Gomi K, et al. Stereotactic hypofractionated high-dose irradiation for stage I NSCLC. Cancer. 2004;101: 1623-31.

5. Fernando HC, De Hoyos A, Landrenau RJ, Gilbert S, Gooding WE, Buenaventura PO, et al. Radiofrequency ablation for the treatment of NSCLC in marginal surgical patients. J Thorac Cardiovasc Surg. 2007;129:639-44.

6. Pennathur A, Luketich JJ, Abbas G, Chen M, Fernando HC, Gooding WE, et al. Radiofrequency ablation for the treatment of stage I NSCLC in high risk patients. J Thorac Cardiovasc Surg. 2007;134:857-64.

7. Simon CJ, Dupuy DE, DiPetrillo TA, Safran HP, Grieco CA, Ng T, et al. Pulmonary radiofrequency ablation: Long term safety and efficacy in 153 patients. Radiology. 2007;243:268-75.

8. Sano Y, Kanazawa S, Gobara H, Mukai T, Hiraki T, Hase S, et al. Feasibility of percutaneous radiofrequency ablation for intrathoracic malignancies. Cancer. 2007; 109:1397-405

9. Nguyen CL, Scott WJ, Young NA, Rader T, Giles LR, Goldberg M. Radiofrequency ablation of primary lung cancer: results from an ablate and resect pilot study. Chest. 2005;128:3507-11.

10. Ginsberg RJ, Rubenstein LV. Randomized trial of lobectomy versus limited resection for T1N0 NSCLC. Lung Cancer Study Group. Ann Thorac Surg. 1995;60: 615-22.

11. El-Sherif A, Gooding WE, Santos R, Pettiford B, Ferson PF, Fernando HC, et al. Outcomes of sublobar resection versus lobectomy for stage I non-small cell lung cancer: a 13-year analysis. Ann Thorac Surg. 2006;82:408-16.
12. Bradley JD, Wahab S, Lockett MA, Perez CA, Purdy JA. Elective nodal failures are uncommon in medically inoperable patients with stage I non-small-cell lung carcinoma treated with limited radiotherapy fields. Int J Radiat Oncol Biol Phys. 2003;56:342-7.

13. Timmerman R, McGarry R, Yiannoutsos C, Papiez L, Tudor K, DeLuca J, et al. Excessive toxicity when treating central tumors in a phase II study of stereotactic body radiation therapy for medically inoperable early-stage lung cancer. J Clin Oncol. 2006;24:4833-9.

14. Guckenberger M, Heilman K, Wulf J, Mueller G, Beckmann G, Flentje M. Pulmonary injury and tumor response after stereotactic body radiotherapy (SBRT): results of a serial follow-up CT study. Radiother Oncol. 2007;85:435-42.

15. Zhu JC, Yan TD, Morris DL. A systematic review of radiofrequency ablation for lung tumors. Ann Surg Oncol. 2008;15:1765-74.

16. Thanos L, Mylona S, Pomoni M, Athanassiadi K, Theakos N, Zoganas. L et al. Percutaneous radiofrequency thermal ablation of primary and metastatic lung tumors. Eur J Cardiothorac Surg. 2006;30:797-800.

17. Yasui K, Kanazawa S, Sano Y, Fujiwara T, Kagawa S, Mimura H, et al. Thoracic tumors treated with CT-guided RFA: initial experience. Radiology. 2004;231: 850-7.

18. Yamamoto A, Nakamura K, Matsuoka T, Toyoshima M, Okuma $T$, Oyama Y, et al. Radiofrequency ablation in a porcine lung model: correlation between CT and histopathologic findings. AJR Am J Roentgenol. 2005; 185:1299-306.

19. Tominaga J, Miyachi H, Takase K, Matsuhashi T, Yamada T, Sato A, et al. Timerelated changes in computed tomographic appearance and pathologic findings after radiofrequency ablation of the rabbit lung: preliminary experimental study. J Vasc Interv Radiol. 2005; 16:1719-26.

20. Dupuy DE, DiPetrillo T, Gandhi S, Ready N, Ng T, Donat W, et al. Radiofrequency ablation followed by conventional radiotherapy for medically inoperable stage I NSCLC. Chest. 2006;129:738-45.

21. VanSonnenberg E, Shankar S, Morrison PR, Nair RT, Silverman SG Jaklitsch MT, et al. Radiofrequency ablation of thoracic lesions: part 2, initial clinical experience-technical and multidisciplinary considerations in 30 patients. AJR Am J Roentgenol. 2005;184:381-90.

22. Sienel W, Dango S, Kirschbaum A, Cucuruz B, Horth W, Stremmel C, et al. Sublobar resections in stage IA non-small cell lung cancer: segmentectomies result in significantly better cancer-related survival than wedge resections. Eur J Cardiothorac Surg. 2008;33:728-34.

23. White RR, Avital I, Sofocleous CT, Brown KT, Brody LA, Covey A, et al. Rates and patterns of recurrence for percutaneous radiofrequency ablation and open wedge for solitary colorectal liver metastasis. J Gastrointest Surg. 2007;11: 256-63.

\section{Discussion}

Dr Neil A. Christie (Pittsburgh, $\mathrm{Pa}$ ). Lanuti and colleagues at Massachusetts General Hospital should be commended for the execution of the study. They are first to be commended for having thoracic surgeons participating both in the selection of these patients and also in the application of the treatment, because it is important that surgeons be involved. They are second to be commended both for carefully evaluating their results and for presenting them now. These newer ablative therapies, such as RFA and stereotactic radiosurgery, are being widely promoted by radiologists and radiation oncologists as alternative nonoperative but potentially curative therapies for early-stage lung cancer. Earlier this week, Dr Roth presented a multi-institutional study in which patients with operable early-stage lung cancer were randomly assigned to undergo either surgery or ablative therapy with stereotactic radiosurgery for lung cancers as large as $5 \mathrm{~cm}$ in diameter. So I think that as people are starting to propose these therapies as curative therapy in operative candidates, it is important that we evaluate them and determine their limitations.

In his presidential address at this meeting, Dr Craig Miller emphasized the importance to cardiothoracic surgeons of training and participating in such novel nonsurgical therapies. He proposed 
a revision to the cardiac training program that would allot almost equal time to surgical training and to novel, catheter-based therapy. At the University of Pittsburgh, we have a hybrid operating room where we do procedures such as RFA, and it is an integral part of our training program. I think that it is highly likely in the future that there will be a subset of patients with early-stage lung cancer who, after careful staging, will receive adequate treatment with nonsurgical ablative therapy instead of surgery. For all these reasons, this is a timely report.

The study demonstrated that the therapy was safely administered. There were no deaths, hospitalization was less than 24 hours, and chest tube placement was required in only $8 \%$ of patients. It also demonstrated maintenance of pulmonary function after therapy, and 3-year progression-free survival was 58\%. Overall, however, there was a $31.5 \%$ local recurrence rate, despite a relatively small mean tumor diameter of $2 \mathrm{~cm}$. In fact, tumors larger than 3 $\mathrm{cm}$ had a $50 \%$ local recurrence rate.

Dr Lanuti, you have discussed the difficulty in assessing these patients in terms of response to therapy and also recurrence rate, and that brings me to my first question. Please comment in a little more detail on how you have addressed posttreatment follow-up? You did use CT scans and PET scans. Did you find concordance between those two studies, and to what extent did you confirm your suspected recurrence with needle biopsy?

Dr Lanuti. All recurrences were confirmed by imaging studies and, on a single occasion, transthoracic needle biopsy. Methods of local recurrence detection included diagnostic CT and fluorodeoxyglucose PET, with relative comparisons among the studies with time. We found 9 of the 12 recurrences in less than 6 months. I think that CT and PET alone are not as valuable as they are together. Patients did not routinely undergo biopsy to confirm the radiographically determined recurrence. No other modalities were used for detection of local recurrence.

Dr Christie. Our experience at Pittsburgh with both RFA and stereotactic radiosurgery has been that postoperative imaging can be hard to interpret. We too have been using PET and CT, and often we will take the first posttreatment study as a baseline to compare other studies. We have been liberal with the use of biopsy, however, especially as we are trying to ascertain what the imaging means.

For my second and final question, I noted that fully a third of the patients did not have successfully local control. Do you have any ideas in terms of how either your technique or the technology might allow us to improve those outcomes? Thank you for allowing me to review your article.

Dr Lanuti. Thank you, Dr Christie, for your comments. I will address your last question; that is, why do we have such a high recurrence rate, and where does that stand among the other observational studies? In fact, the recurrence rate in our study falls within the range observed $(3 \%-42 \%)$. Three of the local recurrences were tumors larger than $3 \mathrm{~cm}$. We learned as the experience matured that the Covidien cluster electrode has clear size limitations. The limitations of RFA electrode technology are tumor size, proximity to blood vessels (because they are heat sinks), and the central nature of the tumor. Although we have treated a single central tumor with RFA, in general, the tumors selected for this modality have been peripheral. We had 2 local recurrences resulting from a procedure-related pneumothorax that allowed the lung to move away from the electrode, because this particular device doesn't have the anchoring tines present in the Boston Scientific electrode. Of note, we had 2 late recurrences, one at 12 months and one at 17 months. The 17-month recurrence was in a patient with previous lung cancer who refused surgery. His treatment failed at 17 months after RFA, and he ultimately went to surgery for a salvage right upper lobectomy. His final pathologic report showed a 1-cm residual adenocarcinoma (T1N0) with extensive hyaline fibrosis consistent with treatment effect.

In summary, our patient population was a heterogeneous group, similar to all the observational studies previously published. Careful patient selection and understanding the limitations of the technology will ultimately reduce the recurrence rate. Although our study population was small, analysis suggests that tumors larger than $2 \mathrm{~cm}$ are more likely to recur locally after RFA with the Covidien cluster electrode.

Dr Bryan Fitch Meyers (St Louis, Mo). Dr Lanuti, there is a direct overlap in the indications for this with stereotactic radiation therapy If I were a patient with inoperable disease, it would just seem more appealing to get three doses of $20 \mathrm{cGy}$ as an outpatient rather than having something stuck in my chest. Could you tell me what the selling points are to a patient to have RFA rather than stereotactic radiation therapy?

Dr Lanuti. That is a good, pertinent, germane question. It is easy at Massachusetts General in Boston, because we don't have any of these CyberKnife or stereotactic radiosurgery programs, so often we would have to refer them to another institution. That is an easy answer. Really, though, you are absolutely right; it is a competitive modality for surgery. I would say that stereotactic radiosurgery does require some interventions. Fiducial markers have to be placed bronchoscopically, so that it is a procedure. It is three separate treatments, although relatively benign, as opposed to RFA, which is a single treatment and then follow-up with multiple scans and a 23-hour hospital stay. The late complications of stereotactic radiosurgery in North America relative to Japan as it is emerging is that the central lesions have much more likelihood of radiation pneumonitis and bronchial stenosis, and probably Dr Christie could comment more than I about their own experience. But there are limitations. So side by side, these modalities would have to be compared. I think they don't have to be considered mutually exclusive but actually potentially complementary; RFA is good for central necrosis, whereas these external beam radiotherapy techniques are good for peripheral type treatment. 